\title{
NOTE ON NAMES
}

With the exception of the names of authors cited whose texts were originally written in English or other European languages, or who use another convention in their professional profiles, all East Asian names follow the East Asian convention, in which family names precede given names. 

ELECTRIFIED VOICES 
